\title{
3D characterization of microstructured poly(methacrylic acid) thin films via Mach-Zehnder interference microscopy
}

\author{
S. Letsche ${ }^{\mathrm{a}, \mathrm{b}}$, P. Jacobs ${ }^{\mathrm{c}}$, M. Pluntke ${ }^{\mathrm{d}}$, S. Tränkle ${ }^{\mathrm{a}, \mathrm{b}}$, H. Gong ${ }^{\mathrm{a}, \mathrm{b}}$, O. Marti ${ }^{\mathrm{d}}$, D.M. Mahlmann ${ }^{\mathrm{c}, *}$, \\ P. Loosen ${ }^{\mathrm{e}}$, D. Volkmer ${ }^{\mathrm{a}, \mathrm{b}, * *}$
}

\footnotetext{
a Augsburg University, Institute of Physics, Chair of Solid State Chemistry, Universitätsstrasse 1, D-86159 Augsburg, Germany

${ }^{\mathrm{b}}$ Ulm University, Institute of Inorganic Chemistry II, Materials and Catalysis, Albert-Einstein-Allee 11, D-89081 Ulm, Germany

' Incelltec GmbH, Steinbachstrasse 15, D-52074 Aachen, Germany

${ }^{\mathrm{d}}$ Ulm University, Institute of Experimental Physics, Albert-Einstein-Allee 11, D-89081 Ulm, Germany

e Technologie Optischer Systeme (TOS), RWTH Aachen University, Steinbachstrasse 15, D-52074 Aachen, Germany
}

\section{Introduction}

In the last decade research in the field of polymer brushes has gained significant technological interest. In particular polymer brush thin films were developed for applications in micro-electronics [1] and biotechnology including anti-fouling coatings [2], biosensors, medical implants and templates for tissue engineering and biomedical materials [2-4]. Polymer brushes are polymer chains, tethered by one end to a surface or interface. At high grafting density, these polymers are forced to stretch away from the surface so as to avoid overlapping [5]. Micropatterned polymer films gained special attraction because they are useful tools serving to control and to confine cell adhesion on solid surfaces [6], to immobilize proteins locally [7] or to direct the nucleation and inclusion of inorganic materials $[8,9]$.

\footnotetext{
* Corresponding author. Tel.: + 492418906 172; fax: + 492418906121

** Correspondence to: D. Volkmer, Augsburg University, Institute of Physics, Chair of Solid State Chemistry, Universitätsstrasse 1, D-86159 Augsburg, Germany. Tel.: +49 821598 3006; fax: +498215985955.

E-mail addresses: d.mahlmann@incelltec.de (D.M. Mahlmann), dirk.volkmer@physik.uni-augsburg.de (D. Volkmer).
}

Among other lithographic methods (microcontact printing [10], electron beam [11], laser interference [12] and nanoimprint lithography [13]) photolithography offers a simple route toward microstructured polymer brushes. The surface topography of polymer brush thin films can be precisely controlled by projecting UV-light through a photomask on the monolayer of a photo-labile polymerization initiator anchored to the substrate surface, followed by polymerization which occurs exclusively in the non-irradiated areas. A huge variety of chemically different monomers can be utilized in the subsequent polymerization step, for which different techniques have been developed such as cationic and anionic [14-16], ring-opening polymerization [17], ring opening metathesis polymerization [18], atom transfer radical polymerization [19,20], nitroxide-mediated radical polymerization [21], or reversible addition fragmentation chain transfer polymerization [22,23]. The geometry of (charged) polyelectrolyte polymer brushes is responsive to environmental conditions such as humidity, electrolyte concentration, and local pH-value (i.e. they can swell, collapse, etc.) [24]. Additionally, the quality and height of polymer brushes depend on different polymerization parameters (irradiation intensity, purity of the monomer, polymerization time, etc.). The brush thickness typically ranges from 
several nanometers (collapsed brush state, frequently observed after prolonged drying in air) to a few microns (swollen state, commonly noted upon exposing films to aqueous solution). It is essential to characterize the geometry of transparent polymer films (homogeneity of the polymerized surface and structure shapes) prior to further processing steps, because samples with structure defects or a large amount of impurities may lead to low quality products and high rates of lost output.

When micropatterned polymer brushes are fabricated as coatings of biomedical implants or cell culture substrates a challenging problem is to check their structural integrity and uniformity over extended areas (at the $\mathrm{cm}^{2}$ scale), for the purpose of which fast, reliable and non-destructive height sampling techniques are required.

The most common film thickness measuring techniques for micropatterned surfaces can be formally divided into contact and non-contact methods. Stylus profilometry and atomic force microscopy (AFM) are based on the mechanical (contact) sampling of surfaces. Both methods are very accurate in vertical (off-plane) direction (theoretical resolution $\leq 1 \AA$ ). While stylus profilometers provide a horizontal resolution (in-plane direction) of typically $2 \mu \mathrm{m}$ and more (depending on stylus diameter), AFMs commonly deliver resolutions down to several nanometers. However, for high resolution sampling, these methods suffer from long measurement times due to the mechanical scanning operation. Moreover the scanning area for commercial AFM devices is restricted to typically $100 \mu \mathrm{m}^{2}$. Another disadvantage of contact sampling techniques is the possible manipulation or even damage of soft-matter samples.

Confocal microscopy is a scanning method that provides a contactless detection of surface structures. This technique can be operated with focused acoustic (ultrasonic) or optical waves (scanning acoustic microscopy (SAM) or confocal laser scanning microscopy (CLSM)) and allows to sample extended surface areas ( $\mathrm{mm}^{2}$ range) in a relatively short time. However the disadvantage is the limited resolution due to the Abbe diffraction limit $(\mathrm{x}-, \mathrm{y}-, \mathrm{z}-$ resolution: a few $100 \mathrm{~nm}$ ). This limit can be overcome with near-field scanning optical microscopy (NSOM/SNOM). Here, the lateral resolution is determined only by the aperture of the probe, which can be minimized down to $20 \mathrm{~nm}$. The disadvantage of this method is the restricted scanning area, as the technical basis of this microscope is similar to the one of AFM.

Classical ellipsometry measures the change in the polarization state of light reflected from the sample surface and is often used for routine height analysis of thin films. Imaging ellipsometry offers a fast and precise optical method for determining film thickness in two dimensions (vertical resolution (i.e. off-plane direction): $1 \mathrm{~nm}$, horizontal resolution (i.e. in-plane direction): $2 \mu \mathrm{m}$, applicable sample area: up to several $100 \mu \mathrm{m}$ ).

However, most commonly optical profilometers are routinely used to measure laterally resolved film thicknesses. They are usually based on the interference of waves having passed through different optical paths. For laser light sources, usually the phase difference between the object wave and a reference wave is detected by measuring the intensity of the interference fringes while the phase difference between the two interfering beams is changed in a known manner. Typical setups include Michelson, Mirau or Mach-Zehnder interferometers. These interferometers are commonly equipped with either laser or white light sources.

The basic difference of the Mach-Zehnder interferometer technique (MZI) in this experiment to common ones is the combination of transmitted light microscopy and Mach-Zehnder interferometry. While the lateral resolution is refraction limited as in any light microscope, the phase resolution for measuring the optical path difference (OPD) between two coherent light waves achieves an accuracy up to $1 \mathrm{~nm}$ depending on the signal-to-noise ratio of the camera used. The measured area depends on the magnification and the numerical aperture of the objectives and can be increased with motorized microscope sample holders. As Mach-Zehnder interferometry is not restricted to microscope optics, the set-up can be used with a telecentric objective for macro photography that allows to image areas on a centimetre scale with a lower spatial resolution but still with a high phase resolution. Because both setups require a high lateral and phase resolution, the use of a white light source is necessary to achieve the best trade-off between these two kinds of resolutions. Laser sources have a higher spatial and temporal coherence that causes coherent noise (speckles, etc.) in the recorded images. White light requires a higher accuracy in the adjustment of the interferometer set-up but leads to unblurred images of the object with high resolution (Table 1).

Since fast quality controls of micropatterned polymer brushes are crucial for their efficient application, we developed a reliable method that can be routinely applied for 3D film thickness characterization. Based on our discussion above, we concluded that the development of a technique that combines highly accurate height measurements with large lateral sampling areas at a fast sampling rate is necessary. Here we present the Mach-Zehnder interferometer which possesses these features and produces accurate results for micropatterned polymer brush thin films if compared with traditional methods (atomic force microscopy, white light profilometry). MZI allows the precise measurement of the optical density of transparent thin films in transmitted light from which the film thickness and the refractive index can be evaluated.

Table 1

Comparison of techniques for 3D surface characterization.

\begin{tabular}{|c|c|c|c|c|c|c|c|}
\hline & Contact methods & & Optical methods & & & & \\
\hline $\begin{array}{l}\text { Method } \\
\text { feature }\end{array}$ & $\begin{array}{l}\text { Atomic force } \\
\text { microscopy [a] }\end{array}$ & $\begin{array}{l}\text { Stylus } \\
\text { profilometry [b] }\end{array}$ & $\begin{array}{l}\text { Imaging } \\
\text { ellipsometry [c] }\end{array}$ & SNOM $[d, e]$ & CLSM [f] & WIM [g] & $\begin{array}{l}\text { Mach-Zehnder } \\
\text { interferometry [h] }\end{array}$ \\
\hline $\begin{array}{l}\text { Measurement } \\
\text { duration }\end{array}$ & Several minutes/hours & $\begin{array}{l}\text { Several minutes/ } \\
\text { hours }\end{array}$ & A few seconds* & $\begin{array}{l}\text { Several minutes/ } \\
\text { hours }\end{array}$ & $\begin{array}{l}\text { A few } \\
\text { seconds }\end{array}$ & A few seconds/minutes & A few seconds* \\
\hline Accuracy & $<1 \AA$ & Max. $1 \AA$ & $1 \mathrm{~nm}$ & $10 \mathrm{~nm}$ & $0.1 \mathrm{~nm}$ & $\begin{array}{l}<1 \AA \text { (but then with small sample } \\
\text { size and speed limitation) }\end{array}$ & $1 \mathrm{~nm}$ \\
\hline $\begin{array}{l}\text { Lateral } \\
\quad \text { resolution }\end{array}$ & A few nm & $2 \mu \mathrm{m}$ & $2 \mu \mathrm{m}$ & $\geq 20 \mathrm{~nm}$ & $150 \mathrm{~nm}$ & A few hundreds of $\mathrm{nm}$ & $250 \mathrm{~nm}$ \\
\hline $\begin{array}{l}\text { Applicable } \\
\text { sample size }\end{array}$ & $100 \mu \mathrm{m} \times 100 \mu \mathrm{m}$ & Up to $150 \mathrm{~mm}^{2}$ & $400 \mu \mathrm{m} \times 400 \mu \mathrm{m}$ & $100 \mu \mathrm{m} \times 100 \mu \mathrm{m}$ & $\begin{array}{l}\text { Several } \\
10 \mathrm{~mm}^{2}\end{array}$ & Up to $20 \mathrm{~mm}^{2}$ & Up to several $\mathrm{cm}^{2}$ \\
\hline
\end{tabular}

* Depends on the availability of the refractive index.

[a] Specifications of the supplier Agilent Technologies, USA; http://cp.literature.agilent.com/litweb/pdf/5989-6405EN.pdf

[b] Specifications of the supplier Veeco Instruments Inc., USA; http://www.bruker-axs.de/uploads/tx_linkselectorforpdfpool/Dektak-150_Stylus_Profilometer_brochure.pdf.

[c] Specifications of the supplier Accurion GmbH, Germany; http://www.accurion.com/download/tech_spec_nanofilm_ep3bam_k .pdf.

[d] E. Betzig, J. K. Trautman, Science 257 (1992) 189.

[e] L. Schermelleh, R. Heitzmann, H. Leonhardt, J. Cell Biol. 190 (2010) 165.

[f] Specifications of the supplier Sensofar-Tech, S.L., Spain; http://www.sensofar.com/products/products_plu4300.html.

[g] Specifications of the supplier Zygo Corporation, USA; http://www.zygo.com/?/met/profilers/ (Series 7000)

[h] H. Beyer, Interferenzmikroskopie (Durchlicht), In: Handbuch der Mikroskopie, VEB Verlag Technik Berlin, 1973. 


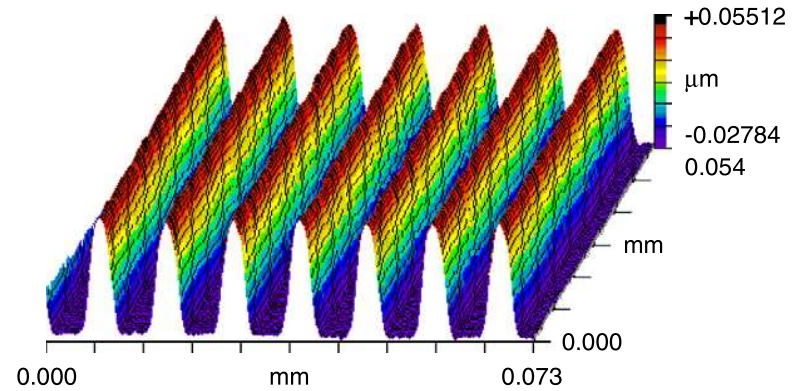

Fig. 1. Topography of the polymer brushes of sample 10_30_1 measured with WIM technique. Typical height is $70 \mathrm{~nm}$ with spacing of $10 \mu \mathrm{m}$.

In order to quantify the structure of micropatterned polymer brushes it is important to differentiate between inhomogeneities in height and local variations of optical densities in the bulk material. As the structuring and polymerization process may lead to both kinds of variations MZI is the only analytical technique that can give reliable and independent information about both parameters.

\section{Experimental details}

\subsection{Fabrication of micropatterned polymer brushes}

Polyelectrolyte poly(methacrylic acid) (PMAA) brushes were prepared on glass substrates by surface-initiated atom transfer radical polymerization of sodium methacrylate, according to a technique described in [7]. For micropatterning, the cleaned glass slides (commercially available round glass microscopy slides, Menzel Gläser, diameter: $18 \mathrm{~mm}, 0.17 \mathrm{~mm}$ thick, borosilicate) were coated with UVlight sensitive 3-(2-bromoisobutyramido)propyl(trimethoxy)silane (initiator) molecules [25]. They were irradiated through a quartz mask with a DUV Hamamatsu Photonics LC8 source (irradiation wavelength $\lambda=250 \mathrm{~nm}$ ). In this process the initiator molecules were selectively inactivated by UV irradiation such that the ensuing polymerization could occur only in the non-irradiated areas. In order to produce polymer brushes with varying pattern size, we designed a photo mask which is comprised of several fields with periodic stripe patterns of different sizes $(10 \mu \mathrm{m}, 20 \mu \mathrm{m}, 40 \mu \mathrm{m})$. For the fabrication of polymer brush thin films with different thicknesses, we varied the polymerization time (10 $\mathrm{min}, 20 \mathrm{~min}$ and $30 \mathrm{~min}$ ). In the following section the samples are labeled according to the following notation: (stripe size)_(polymerization time)_(entry number of sample). All samples were annealed in air for $2 \mathrm{~h}$ at $100^{\circ} \mathrm{C}$ employing a heating plate. Throughout the manuscript the AFMheight of the PMAA brushes represents the average of the two measured heights per sample.

\section{Reference measurements}

The polymer film thickness was determined by two conventional methods, white light profilometry (WIM, manufacturer: Zygo, model: New View 200, analysis software: OMP-0407 C) and atomic force microscopy.

The Zygo New View was used with a $50 \times$ Mirau objective with a numerical aperture of 0.55 that allows a field of view of $140 \times 110 \mu \mathrm{m}$. a)

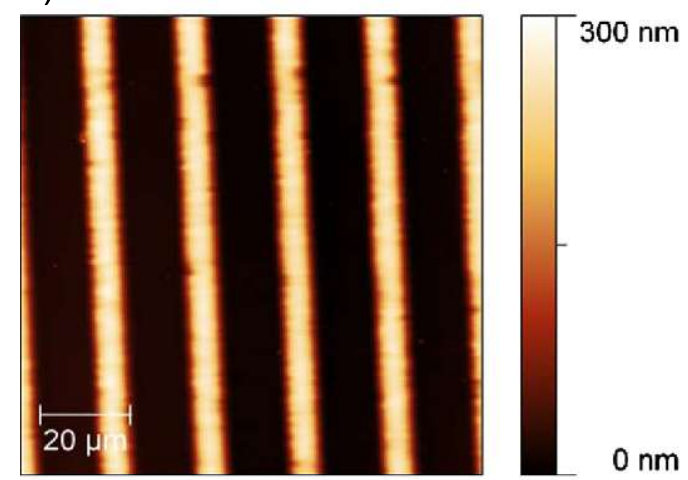

b)

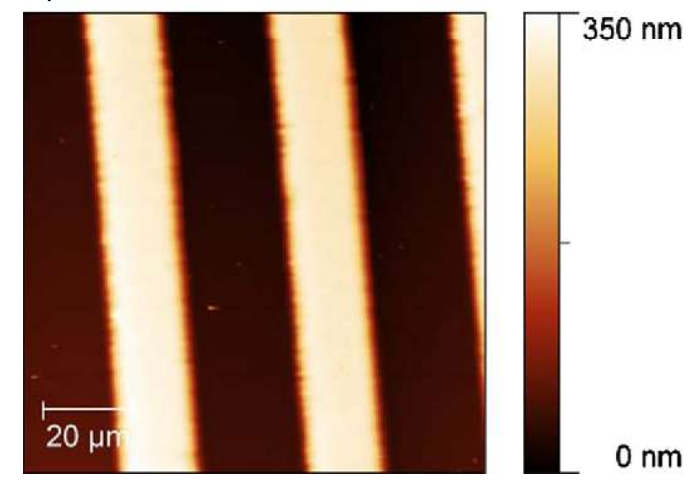

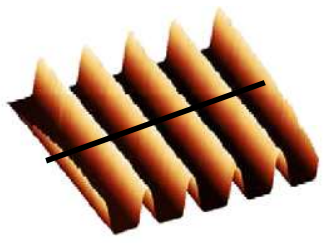
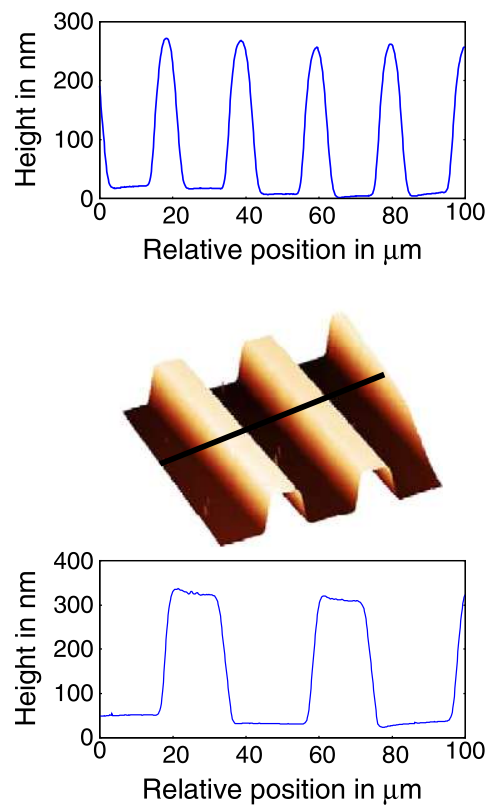
The accuracy in height measurements is given by the manufacturer with less than $1 \mathrm{~nm}[26]$.

A 3D surface plot of sample 10_30_1 as result of a WIM measurement is given as an example in Fig. 1. The shape of the polymer brushes is in good agreement with the measurements performed with AFM and MZI. The WIM measurement has been performed over three different brushes of a single cross-section. The standard deviation of height measurements was between $0.8 \mathrm{~nm}$ and $3.6 \mathrm{~nm}$ for different sampling areas.

AFM measurements have been performed with an Agilent 5500 Scanning Probe Microscope operated in the alternating contact (AC) AFM mode ("tapping mode") at room temperature. Olympus OMCCAC160TS Si-cantilevers with a typical tip-radius of $\sim 10 \mathrm{~nm}$, a typical spring constant of $42 \mathrm{~N} / \mathrm{m}$ and a typical resonance-frequency of $340 \mathrm{kHz}$ were used. The polymer brushes were scanned at two different positions per sample in air. Each measurement took about $10 \mathrm{~min}$. The film thickness was determined by applying a fitting routine to the topography profiles, implemented in the data analysis software 'Gwyddion' [27]. Fig. 2 shows typical AFM topographies, 3D projections and height profiles of two micropatterned PMAA brush samples in the dry state with pattern periodicities of $10 \mu \mathrm{m}$ and $20 \mu \mathrm{m}$, respectively (both samples obtained after $30 \mathrm{~min}$ polymerization time).

Within the sampled areas, the micropatterned polymer brushes are uniform in height and have well defined shapes. The resolution in z-direction of a calibrated AFM scanner is smaller than $1 \AA$. Thus the AFM height measurements are very accurate. The fitting routine used for the analyses of the topographies gives a height uncertainty of about $\pm 4 \%$. Deviations throughout the sample due to an inhomogeneous polymerization process are possible but were not observed in the sampled areas.

The refractive index was measured with an Imaging Elli 2000 Ellipsometer (Nanofilm Technologie GmbH, Göttingen, Germany) equipped with a motorized goniometer for an accurate selection of the incidence angle. The measurement was operated with a $532 \mathrm{~nm}$ $\mathrm{Nd}$ :YAG laser at the incidence angle of $50^{\circ}$. Polymer brush samples prepared on glass cover slides were put on a computer controlled $\mathrm{x} / \mathrm{y} /$ $\mathrm{z}$-stage where the sample position and tilt could be precisely controlled. The ellipsometer is a single wavelength nulling instrument with a polarizer-compensator-sample-analyzer configuration in which a linear polarizer $(\mathrm{P})$ and a quarter-wave plate $(\mathrm{C})$ yield an elliptically polarized incident beam. The polarized beam is reflected from the sample surface, gathered into the analyzer (A) and imaged onto a CCD camera through a long working distance $10 \times$ or $20 \times$ objective. The field of view was about $0.4 \times 0.6 \mathrm{~mm}$ and the lateral resolution about $2 \mu \mathrm{m}$. A region-of-interest (ROI) was selected from the image where the measurement and optical parameter fitting were focused on this area. The ellipsometry measurement was performed by simply changing the $\mathrm{P}, \mathrm{C}$, and A positions to find the null condition (lowest intensity) of the selected area (ROI). The positions that yielded the null condition were then converted into the ellipsometric parameters, $\Delta$ (relative phase shift) and $\Psi$ (relative amplitude ratio). These ellipsometric parameters were further transformed into optical film thicknesses and refractive index using the iElli2000 software, based on the standard electromagnetic treatment for a parallel-layer

Table 2

Resulting refractive index measured by imaging ellipsometry; notation: (polymerization time)_(entry number of sample).

\begin{tabular}{lllll}
\hline Sample name & $\# 1$ & $\# 2$ & $\# 3$ & $\emptyset$ \\
\hline $20 \_2$ & 1.4959 & 1.5000 & 1.4850 & 1.4936 \\
$30 \_1$ & 1.4858 & 1.5170 & 1.4857 & 1.4962 \\
$30 \_2$ & 1.5109 & 1.4932 & 1.4877 & 1.4973 \\
End $\varnothing$ & & & & 1.4957 \\
\hline
\end{tabular}

model [28]. In our case, a three-layer optical model system was used for optical parameter data fitting, i.e. air/polymer brush film/glass cover slide. The refractive index of the glass cover slide at $532 \mathrm{~nm}$ was 1.5255. The polymer brush heights were estimated from AFM measurements. The refractive index of the polymer brush was then calculated with this predetermined thickness data. The measurements and calculations were repeated for several times to ensure a good reproducibility (Table 2 ). The results for the refractive index gave a statistical error of 0.004 .

\section{Method}

\subsection{Mach-Zehnder Interference Microscopy (MZI)}

A white light Mach-Zehnder interferometer is equipped with two wave optically matched microscope optics in each beam path (Fig. 3). The objectives used have a 50x magnification and a numerical aperture of 0.85 . This instrument allows a spatial resolved measurement of the optical density of transparent thin films in transmitted light. A planar light wave, generated by a mercury lamp and a Koehler illumination unit, is divided into an object and a reference wave by wavefront division in a beam splitting prism. By passing through a transparent material the object wave accumulates a phase retardation in comparison to the reference wave caused by the optical density of the object. The two waves interfere in a beam combining prism and an interference pattern can be observed either through an ocular or on a

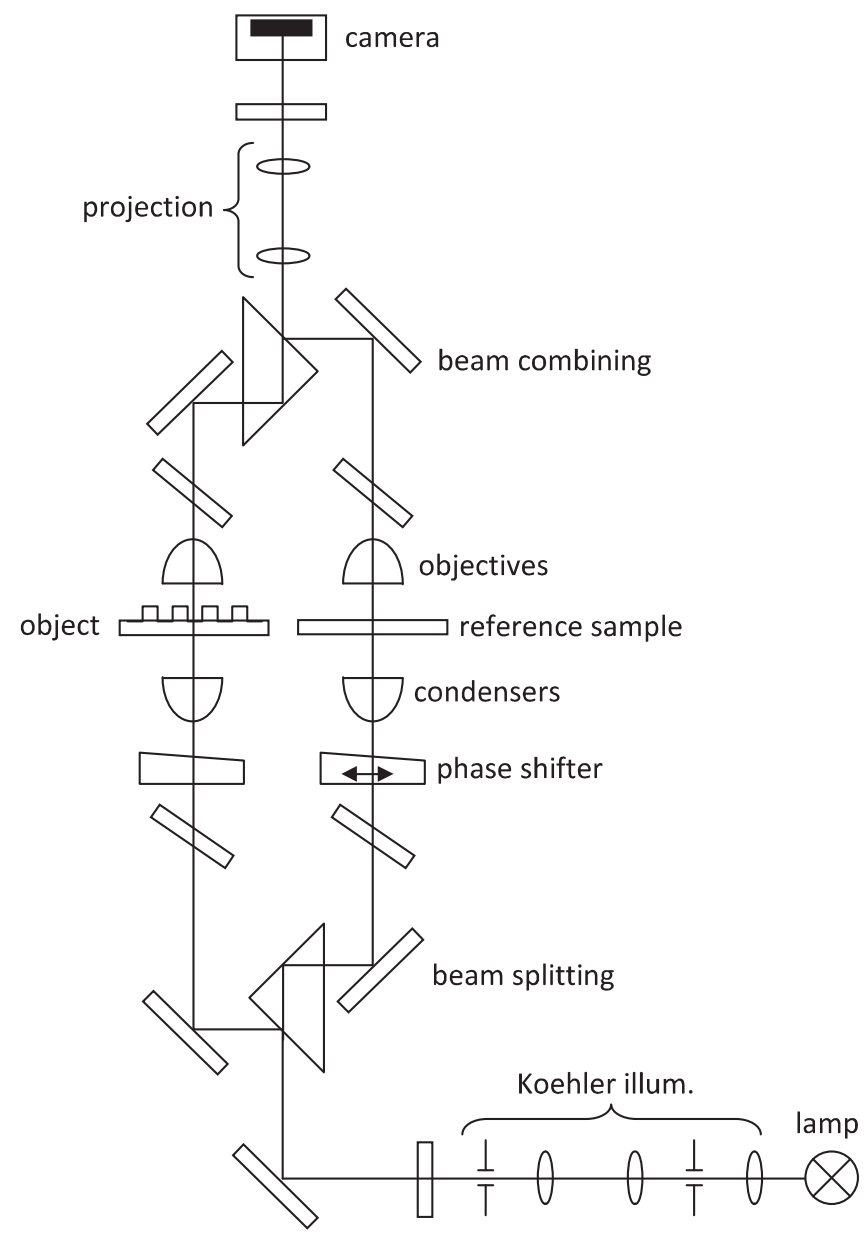

Fig. 3. Schematic drawing of a Mach-Zehnder interference microscope 
camera. By adjusting the angle of incidence between object and reference wave the interference pattern can be adjusted. At steep angles a cosine shaped modulated intensity variation is achieved whereas at flat angles a homogeneous interference field is obtained. The phase retardation of the object wave introduces a curvature in the interference stripe field where the lateral displacement is proportional to the optical path difference (OPD) in relation to the periodicity of the interference pattern. In the homogenous interference field the distribution of the optical density can be determined by applying a phase shifting algorithm $[29,30]$. In this case the object is magnified by the microscope optics, with the specific spatial resolution of the condensers and objectives being used, and the distribution of the optical density is displayed over the whole field of view. In preliminary work the phase resolution of this instrument has been determined to be up to $1 \mathrm{~nm}$ [31].

$O P D=d \cdot\left(n_{O b j}-n_{M e d}\right)$
The OPD is a measure of the thickness of an object as well as the difference between the objects refractive index and the index of the surrounding medium (e.g. air, water or immersion oil). In the interference stripe field the OPD of an object is quantified by measuring the lateral displacement of an interference stripe in the object in comparison to the undisturbed stripe field (Fig. 4). The displacement is gauged with a monochromatic image of the stripe field at a known wavelength and with the same angle of incidence (Fig. 5).

A more accurate calculation can be achieved with a software that has been developed to apply a phase shifting algorithm to MachZehnder Interferometer pictures in a homogeneous interference field $[32,33]$. For this phase shifting algorithm a series of five images is needed. Between two consecutive images the overall phase has to be changed by $\pi / 2$. With this series of pictures the phase distribution or the spatial resulted OPD can be calculated [29].

Starting from the OPD the actual thickness of a specimen and its refractive index can be determined by making at least two measurements with different refractive indices of the embedding medium. The resulting system of equations (ref. Eq. (1)) can be solved

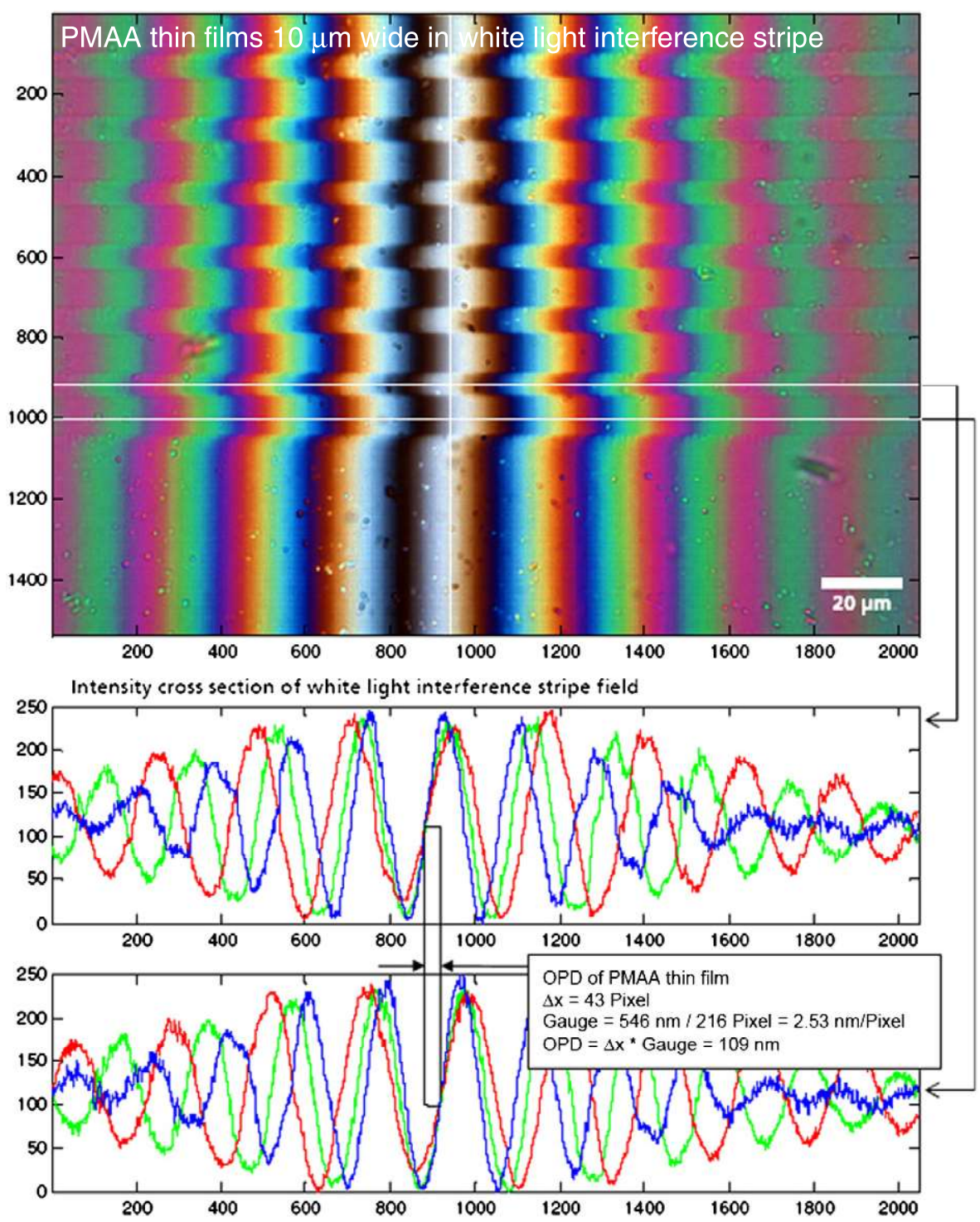

Fig. 4. The PMAA thin films (running horizontal through the picture) cause a displacement of the vertical interference stripes (Sample 10_30_1). The lateral displacement is a direct measure for the OPD of a thin film. After gauging the white light image with a monochromatic image that has the same interference adjustment (Fig. 5 ) but a known wavelength of $546 \mathrm{~nm}$, the lateral displacement can be gauged (box). 


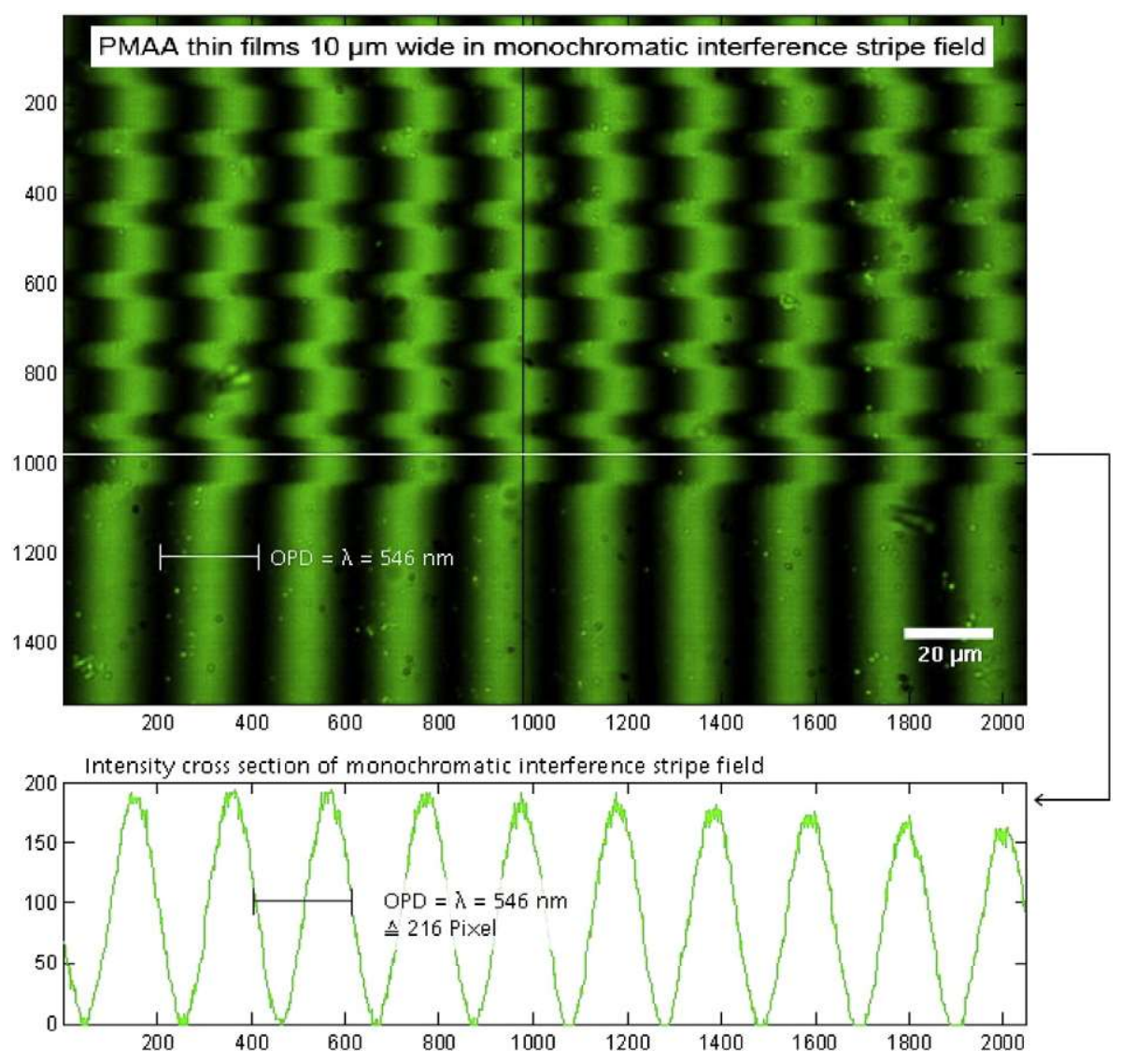

Fig. 5. Same object as in Fig. 4 but with monochromatic filtered light. Due to the physical principle of interference the OPD of two adjacent interference minima is exactly the wavelength of illuminating light. In this case $\lambda=546 \mathrm{~nm}$. Without changing any alignment of the interference conditions, especially the angle of incidence between object and a reference wave, the lateral displacement in Fig. 4 can be gauged.

to either property, i.e. the thickness d, or the refractive index $n_{\mathrm{Obj}}$ of the object.

$d=\frac{O P D_{1}-O P D_{2}}{n_{M e d, 1}-n_{M e d, 2}}$

$n_{\text {Obj }}=\frac{O P D_{1} \cdot n_{\mathrm{Med}, 2}-O P D_{2} \cdot n_{\mathrm{Med}, 1}}{O P D_{1}-O P D_{2}}$

In order to compare the MZI measurements with AFM and WIM the recorded OPD data were analyzed according to the different approaches presented in Eqs. (2) and (3). In a first attempt the height data are directly compared with those determined by other methods using the measured refractive index value by ellipsometry, that the refractive index of the polymer films is about 1.4957, regardless of the different polymerization durations. In a second approach the OPD data of measurements with different embedding media are used to solve the ambiguous Eqs. (2) and (3) and to determine both, height and refractive index. The resulting indices are compared with ellipsometry data as reference method. The second approach appears to be more sophisticated as the polymer thin films interact with its embedding media.

\section{Results and discussion}

All samples were measured with air as the surrounding medium $\left(\mathrm{n}_{\mathrm{D}}=1.0000\right)$. Three of the samples were also examined using water $\left(\mathrm{n}_{\mathrm{D}}=1.3345\right)$ and a specially calibrated mineral oil (Cargille Labs)
$\left(\mathrm{n}_{\mathrm{D}}=1.7000\right)$ as alternative embedding media. At least two measurements with different media are necessary to calculate the height and refractive index of the patterned PMAA brushes without prior knowledge (see Eqs. (2) and (3)). The third measurement is done to enhance accuracy of the obtained results. The optical path difference is extracted out of the phase maps as shown in Figs. 6 and 7. In the vertical cross-section the different trenches can easily be analyzed. For each sample five different trenches were examined. The results are summarized in Table 3. Statistical fluctuations due to the noise of the camera used give rise to a standard deviation of the optical path difference in a given area of about $3 \mathrm{~nm}$ (as can be seen in the horizontal cross section of Fig. 6). Additionally the height of the structures in a given sample varies in the range of $\pm 5 \%$. This is reflected in the standard deviation for the optical path difference measured with the Mach-Zehnder interference microscope.

The resulting refractive index of 1.4957, which was measured with the ellipsometer and which is consistent with the range of values given in [35], was used to calculate the brush heights according to Eq. (1). The resulting heights (MZI) together with comparative data obtained by white light profilometry (WIM) and by atomic force microscopy (AFM) are shown in Table 4.

The WIM-data for samples 10_30_1 and 10_30_2 could not be measured due to insufficient backscattering of reflected light. For better visualization of the results all measurements are plotted in Fig. 8.

It can be derived from the data presented above that all measurement methods give nearly the same results. Obvious deviations might be due to the different measuring sites on the samples 


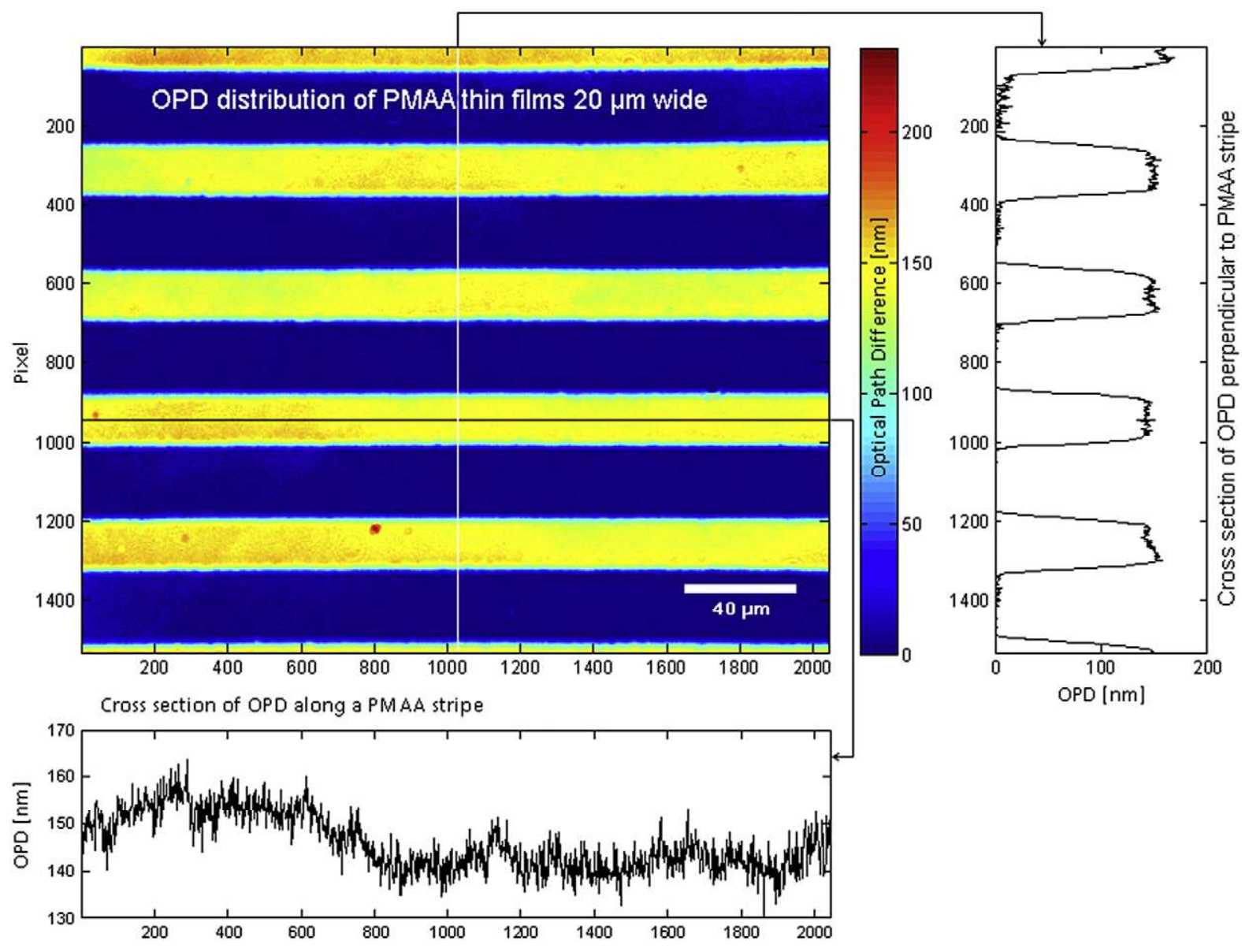

Fig. 6. By capturing a series of interference images in a homogenous interference field [32] the distribution of the OPD can be calculated with a phase shifting algorithm [34]. The results are displayed as a false colour image. The OPD can be determined for any single pixel in the image. The horizontal cross section shows the variation of the OPD in nm along a PMAA thin film. (Higher frequencies are caused by a low signal-to-noise ratio of the used camera.) The vertical cross section in addition to the OPD in nm also shows the shape of the thin films.

as the brush height is varying at least $\pm 5 \%$. Additionally the results from the Mach-Zehnder interference microscope are very sensitive to slight changes in the refractive index, which can also lead to dissimilar results as the refractive index of the various samples might be slightly different. These slight differences in the refractive index might be due to the processing of the material. Generally the refractive index calculated with OPD and WIM heights or AFM heights are similar and are in good agreement with the result received from the ellipsometer. Possible deviations come from the different heights determined by different methods. This also shows that this method is a valueable technique.

It should be noted that without prior knowledge of the refractive index of the used polymers additional measurements can be performed to calculate the local film thickness as well as the refractive index of the samples. For this purpose the optical path difference of three selected samples was measured using different embedding media (air, water and oil) as stated above. The results are summarized in Table 5.

Using Eqs. (2) and (3) the refractive index and the height of the structures can be calculated with $\mathrm{n}$ measurements leading to $\frac{n \cdot(n-1)}{2}$ different combinations.

The calculated values for three distinct samples can be derived from Table 6.

The calculated values for the samples with water as medium show satisfactory values in comparison with the value measured in air. The deviation of the measured values in air is less than $2 \%$. Calculated values for samples 20_10_1 and 10_20_1 show inconsistent results, which might be due to unspecific interactions of the samples with the mineral oil. One type of reaction might be diffusion of mineral oil into the bulk material with subsequent swelling leading to false results. It is shown that when no interactions of the embedding medium with sample material takes place Mach-Zehnder interference microscopy is suitable for measuring the refractive index and the height of existing structures of transparent material.

\section{Conclusions}

In conclusion, we have demonstrated that Mach-Zehnder interference microscopy is an effective tool for the determination of thickness and refractive index of transparent materials, such as micro-structured polymer thin films. The measured film thickness values are in good agreement with the corresponding values obtained from conventional profilometric techniques, namely AFM and WIM. Additional information about the distribution of the refractive index can be gained by the spartially-resolved depiction of the optical path difference. Inhomogeneities and other impurities in the bulk volume can be identified and used to further enhance the polymerization process. 


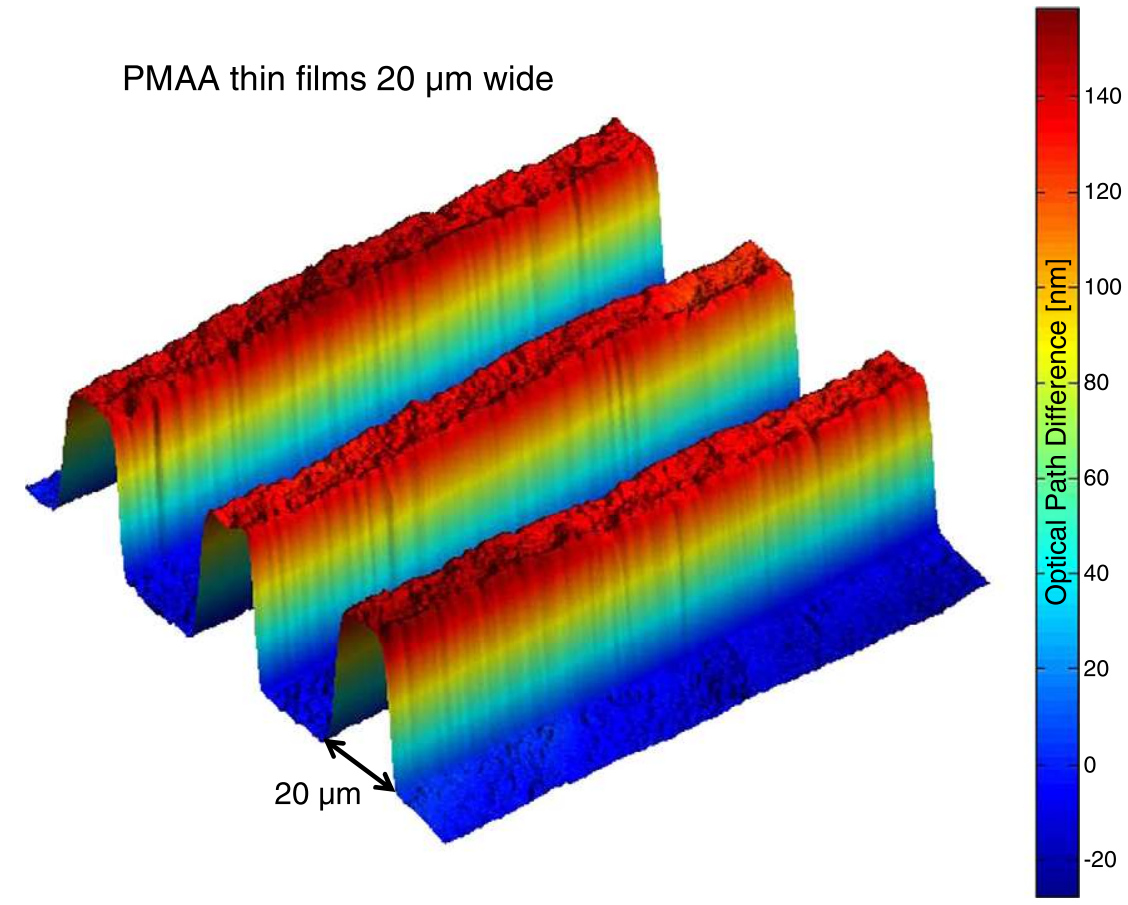

Fig. 7. The OPD distribution of the polymer brushes can be displayed as 3D-image that enables a quantitative measurement of the homogeneity along each stripe.

Table 3

Resulting optical path difference in $\mathrm{nm}$ measured by Mach-Zehnder interferometry; notation: (Stripe size)_(polymerization time)_(entry number of sample).

\begin{tabular}{lllllll}
\hline Sample name & $\# 1$ & $\# 2$ & $\# 3$ & $\# 4$ & \multicolumn{1}{l}{ \#5 } & \multicolumn{1}{l}{$\emptyset$} \\
\hline 10_10_1 & $52 \mathrm{~nm}$ & $46 \mathrm{~nm}$ & $53 \mathrm{~nm}$ & $54 \mathrm{~nm}$ & $49 \mathrm{~nm}$ & $51 \pm 3 \mathrm{~nm}$ \\
20_10_1 & $53 \mathrm{~nm}$ & $53 \mathrm{~nm}$ & $56 \mathrm{~nm}$ & $52 \mathrm{~nm}$ & $54 \mathrm{~nm}$ & $54 \pm 1 \mathrm{~nm}$ \\
10_10_2 & $22 \mathrm{~nm}$ & $23 \mathrm{~nm}$ & $26 \mathrm{~nm}$ & $21 \mathrm{~nm}$ & $21 \mathrm{~nm}$ & $23 \pm 2 \mathrm{~nm}$ \\
20_10_2 & $39 \mathrm{~nm}$ & $36 \mathrm{~nm}$ & $35 \mathrm{~nm}$ & $41 \mathrm{~nm}$ & $41 \mathrm{~nm}$ & $38 \pm 3 \mathrm{~nm}$ \\
10_10_3 & $32 \mathrm{~nm}$ & $32 \mathrm{~nm}$ & $31 \mathrm{~nm}$ & $26 \mathrm{~nm}$ & $33 \mathrm{~nm}$ & $31 \pm 3 \mathrm{~nm}$ \\
20_10_3 & $46 \mathrm{~nm}$ & $56 \mathrm{~nm}$ & $55 \mathrm{~nm}$ & $54 \mathrm{~nm}$ & $54 \mathrm{~nm}$ & $53 \pm 4 \mathrm{~nm}$ \\
10_20_1 & $67 \mathrm{~nm}$ & $75 \mathrm{~nm}$ & $67 \mathrm{~nm}$ & $69 \mathrm{~nm}$ & $67 \mathrm{~nm}$ & $69 \pm 3 \mathrm{~nm}$ \\
20_20_1 & $102 \mathrm{~nm}$ & $99 \mathrm{~nm}$ & $97 \mathrm{~nm}$ & $97 \mathrm{~nm}$ & $105 \mathrm{~nm}$ & $100 \pm 3 \mathrm{~nm}$ \\
10_30_1 & $73 \mathrm{~nm}$ & $78 \mathrm{~nm}$ & $66 \mathrm{~nm}$ & $72 \mathrm{~nm}$ & $67 \mathrm{~nm}$ & $71 \pm 5 \mathrm{~nm}$ \\
20_30_1 & $107 \mathrm{~nm}$ & $117 \mathrm{~nm}$ & $114 \mathrm{~nm}$ & $109 \mathrm{~nm}$ & $115 \mathrm{~nm}$ & $112 \pm 4 \mathrm{~nm}$ \\
10_30_2 & $117 \mathrm{~nm}$ & $124 \mathrm{~nm}$ & $124 \mathrm{~nm}$ & $121 \mathrm{~nm}$ & $123 \mathrm{~nm}$ & $122 \pm 3 \mathrm{~nm}$ \\
20_30_2 & $151 \mathrm{~nm}$ & $147 \mathrm{~nm}$ & $149 \mathrm{~nm}$ & $147 \mathrm{~nm}$ & $149 \mathrm{~nm}$ & $148 \pm 2 \mathrm{~nm}$ \\
\hline
\end{tabular}

Table 4

Comparative data for height measurement using three different measuring techniques $\left(\mathrm{n}_{\mathrm{Med}}=1.4957\right)$.

\begin{tabular}{|c|c|c|c|c|c|}
\hline \multirow[b]{2}{*}{$\begin{array}{l}\text { Sample } \\
\text { name }\end{array}$} & \multicolumn{4}{|c|}{ Height measurements [nm] } & \multirow[b]{2}{*}{$\begin{array}{l}\text { Index calculated } \\
\text { with OPD and } \\
\text { AFM heights }\end{array}$} \\
\hline & WIM & AFM & MZI & $\begin{array}{l}\text { Index calculated } \\
\text { with OPD and } \\
\text { WIM heights }\end{array}$ & \\
\hline 10_10_1 & $82 \pm 3.7$ & $84 \pm 0.1$ & $102 \pm 6.3$ & 1.621 & 1.605 \\
\hline 20_10_1 & $109 \pm 1.0$ & $115 \pm 3.5$ & $107 \pm 2.5$ & 1.491 & 1.467 \\
\hline 10_10_2 & $39 \pm 0.8$ & $42 \pm 4.8$ & $45 \pm 4.6$ & 1.578 & 1.536 \\
\hline 20_10_2 & $74 \pm 1.3$ & $76 \pm 0.1$ & $77 \pm 5.5$ & 1.518 & 1.506 \\
\hline 10_10_3 & $50 \pm 2.0$ & $56 \pm 1.5$ & $62 \pm 5.7$ & 1.610 & 1.546 \\
\hline 20_10_3 & $106 \pm 4.0$ & $116 \pm 8.8$ & $106 \pm 7.9$ & 1.503 & 1.457 \\
\hline 10_20_1 & $109 \pm 1.5$ & $162 \pm 8.7$ & $138 \pm 6.7$ & 1.635 & 1.426 \\
\hline 20_20_1 & $213 \pm 2.5$ & $201 \pm 2.0$ & $200 \pm 6.8$ & 1.468 & 1.498 \\
\hline 10_30_1 & NA & $143 \pm 8.4$ & $142 \pm 9.7$ & NA & 1.497 \\
\hline 20_30_1 & $235 \pm 4.1$ & $233 \pm 7.8$ & $225 \pm 8.4$ & 1.478 & 1.483 \\
\hline 10_30_2 & NA & $249 \pm 1.4$ & $243 \pm 6.0$ & NA & 1.488 \\
\hline 20_30_2 & $270 \pm 3.6$ & $286 \pm 8.2$ & $297 \pm 3.4$ & 1.549 & 1.518 \\
\hline
\end{tabular}

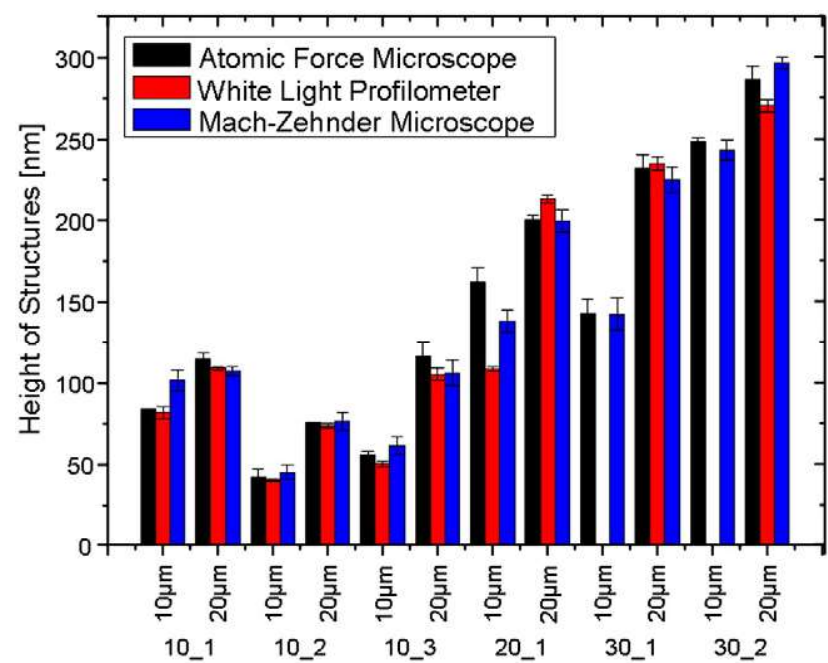

Fig. 8. Comparison of the measured polymer brush height (different samples) using different measuring techniques.

Table 5

Results from MZI-measurements with different embedding media.

\begin{tabular}{llll}
\hline & \multicolumn{3}{l}{ Measured OPD by Mach-Zehnder interferometry [nm] } \\
\cline { 2 - 4 } & Air $\left(\mathrm{n}_{\mathrm{D}}=1.0000\right)$ & Water $\left(\mathrm{n}_{\mathrm{D}}=1.3345\right)$ & Mineral Oil $\left(\mathrm{n}_{\mathrm{D}}=1.7000\right)$ \\
\hline $20 \_10 \_1$ & $51.08 \pm 0.92$ & $14.92 \pm 2.24$ & $-11.72 \pm 0.88$ \\
$10 \_20 \_1$ & $74.58 \pm 4.99$ & $21.68 \pm 1.86$ & $-14.35 \pm 0.68$ \\
$10 \_30 \_1$ & $71.24 \pm 4.82$ & $25.97 \pm 2.20$ & $-26.10 \pm 2.97$ \\
\hline
\end{tabular}

Table 6

Calculated refractive index and film thickness using the results from MZI-measurements with three different embedding media (air, water, oil).

\begin{tabular}{|c|c|c|c|c|c|c|}
\hline & \multicolumn{2}{|l|}{ Air-water } & \multicolumn{2}{|l|}{ Air-oil } & \multicolumn{2}{|l|}{ Water-oil } \\
\hline & Height [nm] & $\mathrm{n}_{\mathrm{D}}$ & Height [nm] & $\mathrm{n}_{\mathrm{D}}$ & Height [nm] & $\mathrm{n}_{\mathrm{D}}$ \\
\hline 20_10_1 & 108.10 & 1.4725 & 89.71 & 1.5694 & 72.89 & 1.5392 \\
\hline 10_20_1 & 158.15 & 1.4716 & 127.04 & 1.5870 & 98.58 & 1.5544 \\
\hline 10_30_1 & 135.31 & 1.5264 & 139.06 & 1.5123 & 142.46 & 1.5168 \\
\hline
\end{tabular}




\section{References}

[1] G.L. Whiting, H.J. Snaith, S. Khodabakhsh, J.W. Andreasen, D.W. Breiby, M.M Nielsen, N.C. Greenham, R.H. Friend, W.T.S. Huck, Nano Lett. 6 (2006) 573.

[2] S. Krishnan, C.J. Weinman, C.K. Ober, J. Mater. Chem. 18 (2008) 3405

[3] J.E. Raynor, J.R. Capadona, D.M. Collard, T.A. Petrie, A.J. García, Biointerphases 4 (2009) FA3.

[4] T. Matsuda, Adv. Polym. Sci. 197 (2006) 67.

[5] S.T. Milner, Science 251 (1991) 905.

[6] R. Iwata, P. Suk-Ink, V.P. Hoven, A. Takahara, K. Akiyoshi, Y. Iwasaki, Biomacromolecules 5 (2004) 2308.

[7] S. Tugulu, A. Arnold, I. Sielaff, K. Johnsson, H.-A. Klok, Biomacromolecules 6 (2005) 1602.

[8] L.M. Bronstein, D.M. Chernyshov, P.M. Valetsky, E.A. Wilder, R.J. Spontak Langmuir 16 (2000) 8221.

[9] S. Joly, R. Kane, L. Radzilowski, T. Wang, A. Wu, R.E. Cohen, E.L. Thomas, M.F Rubner, Langmuir 16 (2000) 1354

[10] Y. Xia, G.M. Whitesides, Langmuir 13 (1997) 2059

[11] I.S. Maeng, J.W. Park, Langmuir 19 (2003) 4519.

[12] F. Yu, F. Mücklich, P. Li, H. Shen, S. Mathur, C.-M. Lehr, U. Bakowsky, Biomacromolecules 6 (2005) 1160

[13] L.J. Gua, Adv. Mater. 19 (2007) 495.

[14] R. Jordan, A. Ulman, J. Am. Chem. Soc. 120 (1998) 243.

[15] R. Advincula, Q. Zhou, M. Park, S. Wang, J. Mays, G. Sakellariou, S. Pispas, N. Hadjichristidis, Langmuir 18 (2002) 8672

[16] R. Advincula, Adv. Polym. Sci. 197 (2006) 107

[17] G. Carrot, D. Rutot-Houzé, A. Pottier, P. Degée, J. Hilborn, P. Dubois, Macromolecules 35 (2002) 8400.

[18] M. Weck, J.J. Jackiw, R.R. Rossi, P.S. Weiss, R.H. Grubbs, J. Am. Chem. Soc. 121 (1999) 4088

[19] K. Matyjaszewski, J. Xia, Chem. Rev. 101 (2001) 2921.
[20] J. Pyun, T. Kowalewski, K. Matyjaszewski, Macromol. Rapid Commun. 24 (2003) 1043.

21] C.J. Hawker, A.W. Bosman, E. Harth, Chem. Rev. 101 (2001) 3661

[22] G. Moad, E. Rizzardo, S.H. Thang, Aust. J. Chem. 58 (2005) 379.

[23] G. Moad, E. Rizzardo, S.H. Thang, Aust. J. Chem. 62 (2009) 1402.

[24] J. Rühe, M. Ballauff, M. Biesalski, P. Dziezok, F. Gröhn, D. Johannsmann, N. Houbenov, N. Hugenberg, R. Konradi, S. Minko, M. Motornov, R.R. Netz, M. Schmidt, C. Seidel, M. Stamm, T. Stephan, D. Usov, H. Zhang, in: R. Jordan (Ed.), Polyelectrolyte brushes, Advances in Polymer Science, vol. 165, Springer Verlag, Heidelberg, 2004, p. 79.

[25] S. Tugulu, A. Arnold, I. Sielaff, K. Johnsson, H.-A. Klok, Biomacromolecules 6 (2005) 1602 .

[26] P. de Groot, X.C. de Lega, J. Kramer, M. Turzhitsky, Appl. Opt. 41 (2002) 4571

[27] http://gwyddion.net/.

[28] R.M.A. Azzam, N.M. Bashara, Ellipsometry and Polarized Light, North-Holland, Amsterdam, The Netherlands, 1977

[29] J. Schwider, R. Burow, K.-E. Elssner, J. Grzanna, R. Spolaczyk, K. Merkel, Appl. Opt 22 (1983) 3421.

[30] K. Creath, in: K. Creath (Ed.), Comparison of phase-measurement algorithms, Surface Characterization and Testing, Proc. SPIE - the International Society for Optical Engineering, vol. 680, 1986, p. 19.

[31] D. Esser, D. Mahlmann, D. Wortmann, J. Gottmann, Appl. Phys. B. 96 (2009) 453

[32] D. Mahlmann, J. Jahnke, P. Loosen, Eur. J. Phycol. 43 (2008) 355.

[33] J. Jahnke, D. Mahlmann, J. Appl. Phycol. 22 (2010) 117.

[34] J. Schwider, R. Burow, K.-E. Elssner, J. Grzanna, R. Spolaczyk, K. Merkel, Appl. Opt 22 (1983) 3421

[35] A.Y. Sankhe, S.M. Husson, S.M. Kilbey II, J. Polym, J. Polym. Sci., Part A: Polym Chem. 45 (2007) 566

L.A. Bimendina, V.V. Roganov, E.A. Bekturov, J. Polym. Sci. Symp. 44 (1974) 65 M. Biesalski, J. Rühe, D. Johannsmann, J. Chem. Phys. 111 (1999) 7029; M. Niwa, T. Mori, N. Higashi, Macromolecules 26 (1993) 1936 